\title{
Caracterização dos sintomas e funcionalidade de idosos com necessidade de cuidados paliativos na Estratégia Saúde da Família
}

\section{Evaluation of symptoms and functionality of elderly people in need of palliative care in the Family Health Strategy}

Emilly Pennas Marciano Marques ${ }^{1}$, Daniela Wosiack da Silva², Fernando Cesar Iwamoto Marcucci ${ }^{3}$, Ligia Maria Facci ${ }^{4}$, Geovana Pizzaia Pretti ${ }^{5}$

1. ORCID: https://orcid.org/0000-0001-7045-9739. Fisioterapeuta. Especialista em Saúde da Família. Universidade Estadual de Londrina, Londrina, Paraná, Brasil.

E-mail: emilly.pennas@gmail.com

2. ORCID: https://orcid.org/0000-0003-3702-2724. Fisioterapeuta. Doutora em Saúde Coletiva. Universidade Estadual de Londrina, Londrina, Paraná, Brasil. E-mail: daniwosiack@hotmail.com

3. ORCID: https://orcid.org/0000-0001-8139-7772. Fisioterapeuta. Doutor em Saúde Coletiva. Hospital Dr. Anísio Figueiredo - Secretaria de Saúde do Paraná, Londrina, Paraná, Brasil.

E-mail: $\underline{\text { ccim@msn.com }}$

4. ORCID: https://orcid.org/0000-0002-6182-8122. Fisioterapeuta. Doutora em Ciências da Saúde. Universidade Estadual de Londrina, Londrina, Paraná, Brasil.

E-mail: ligiafacci@uel.com

5.ORCID: https://orcid.org/0000-0003-3109-9157. Fisioterapeuta. Universidade Estadual de Londrina, Londrina, Paraná, Brasil.

E-mail: geovanapretti@hotmail.com

CONTATO: Autor correspondente: Emilly Pennas Marciano Marques | Endereço: Rua Elias Viscardi, 346 Jd. Santa Rita. Londrina, Paraná, Brasil. Telefone: (43) 996649026 E-mail: emilly.pennas@gmail.com 


\section{RESUMO}

O cuidado paliativo (CP) visa propiciar qualidade de vida aos pacientes com doenças que ameaçam a vida, porém, as características de idosos com esta necessidade na Estratégia Saúde da Família (ESF) ainda são pouco exploradas. O objetivo desse estudo foi identificar e caracterizar os idosos com indicação de CP na ESF. Trata-se de um estudo descritivo exploratório quantitativo, com triagem inicial de 428 idosos. Destes, 15 tiveram indicação de CP e foram avaliados por questionário sociodemográfico e instrumentos padronizados. A maioria dos pacientes eram do sexo feminino, com idade média de 75 anos e apresentavam síndromes demenciais, doenças neurológicas, fragilidade e déficit funcional. Os principais sintomas foram dor, alteração no sono e perda de apetite. As principais demandas de saúde observadas foram preocupação familiar e o manejo da dor. Observou-se que os idosos participantes apresentam condições de fragilidade, dificuldade no manejo dos sintomas e baixa capacidade funcional, impactando sua qualidade de vida.

DESCRITORES: Cuidados Paliativos. Idoso. Atenção Primária à Saúde. Estratégia Saúde da Família.

\section{ABSTRACT}

Palliative care $(P C)$ aims to provide quality of life to patients with life-limiting illness, however, the characteristics of elderly people with this need in the Family Health Strategy (FHS) are still little explored. The objective of this study was to identify and to characterize the elderly with indication of PC in the FHS. This is a quantitative descriptive exploratory study, with initial screening of 428 elderly, of which 15 have indication of PC and were evaluated by a sociodemographic questionnaire and standardized instruments. Most patients were female, with an average age of 75 years and had dementia syndromes, neurological diseases, fragility and functional deficit. The main symptoms were pain, sleep troubles and lack of appetite. The main demands were family worries and difficult in pain management. It was observed that elderly people in need of PC present fragility, difficulty in the management of symptoms and low functional capacity, impacting their quality of life.

DESCRIPTORS: Palliative Care. Aged. Primary Health Care. Family Health Strategy. 


\section{INTRODUÇÃO}

envelhecimento é um processo natural da vida, no qual ocorrem alterações físicas, psíquicas e sociais que podem acontecer com autonomia, independência e qualidade de vida até a morte. Contudo, esse processo é influenciado pelo contexto social, político e econômico em que o indivíduo está inserido, incluindo a redução ou perda desses aspectos ${ }^{1-2}$.

No Brasil, é possível observar a ocorrência de transição demográfica frente ao aumento progressivo no contingente de pessoas idosas e, concomitantemente, transição epidemiológica com maior incidência de Doenças Crônicas Não Transmissíveis (DCNT), caracterizadas por patologias multifatoriais e de longa duração associadas à deterioração progressiva da condição clínica e da qualidade de vida dos indivíduos, com repercussões físicas, psicológicas e sociais, que podem se constituir nas principais causas de morbimortalidade e incapacidade, sendo responsáveis por $72 \%$ das causas de mortes no mundo, principalmente em locais de baixa renda ${ }^{1-3}$.

Todavia, é possível observar as repercussões das DCNT na população brasileira e sua complexidade na assistência à saúde, sobretudo em pessoas idosas, as quais trazem desafios para os diferentes níveis de atenção à saúde. Diante disso, os cuidados paliativos (CP) visam proporcionar aos pacientes, condições que favoreçam a viver com qualidade de vida, controlar os sintomas e promover a autonomia-6.

Cuidado paliativo é definido como:

Cuidado holístico ativo dos indivíduos com graves sofrimentos relacionados com a saúde devido a doença grave e, especialmente, daqueles perto do fim da vida, com o objetivo de melhorar a qualidade de vida dos pacientes, familiares e cuidadores ${ }^{7}$.

A Atenção Primária à Saúde (APS) compreende um modo de atenção à saúde baseado em tecnologias, práticas, métodos e evidências científicas que visam promover a longitudinalidade e integralidade do cuidado ao paciente, por meio de ações preventivas e de promoção de saúde, agregando a família, comunidade e profissionais de saúde ${ }^{8}$. Ambas as áreas compartilham de uma abordagem integral centrada no paciente, mas o perfil dos pacientes e as ações desenvolvidas 
relacionadas aos CP na APS, em particular para a população idosa, ainda são escassamente descritas na literatura no Brasil ${ }^{9}$.

O presente estudo teve como objetivo identificar os idosos com indicação de CP cadastrados na Estratégia Saúde da Família (ESF) que atende uma área de vulnerabilidade social, e caracterizar os aspectos sociodemográficos e clínicos, principais sintomas apresentados, funcionalidade e atendimento ofertado para os pacientes pela Unidade Básica de Saúde (UBS).

\section{MÉTODO}

Trata-se de um estudo descritivo exploratório, de abordagem quantitativa, realizado na área de abrangência de uma UBS de Londrina (PR). A UBS atende a quatro bairros, sendo dois na zona urbana e dois na área periurbana, reconhecidos como áreas de vulnerabilidade social. Além das 4 equipes de Saúde da Família, o serviço conta com equipe do Núcleo Ampliado de Saúde da Família (NASF) da residência multiprofissional em saúde da família e com profissionais médicos da residência médica do município.

Esta pesquisa está vinculada ao projeto de pesquisa intitulado "Inserção da Residência Multiprofissional em Saúde da Família na Rede de Atenção à Saúde do Município de Londrina" (CAAE 98572718.4.0000.523; Parecer 3.028.451) e, respeitou aos princípios éticos contemplados na Resolução 466/2012, sendo realizada com autorização da Secretaria Municipal de Saúde de Londrina (SMS).

Para a realização desta pesquisa foram selecionados indivíduos idosos, com 60 anos ou mais, cadastrados na ESF, residentes na área de abrangência da UBS, com uma população inicial projetada de 565 idosos. A coleta de dados ocorreu de março de 2019 a fevereiro de 2020.

Inicialmente, foi realizada a apresentação do tema em estudo para as equipes da ESF, os objetivos da pesquisa e atividades propostas. Posteriormente, foi solicitado aos Agentes Comunitários de Saúde (ACS) de cada equipe, a indicação dos indivíduos idosos cadastrados em suas microáreas populacionais que apresentassem as condições de saúde pré-estabelecidas mais frequentemente associadas aos $\mathrm{CP}^{10}$.

A partir dos pacientes indicados, todos os selecionados que aceitaram participar da pesquisa mediante consentimento através do Termo de Consentimento 
Livre e Esclarecido (TCLE) foram submetidos à triagem inicial. Indivíduos caracterizados como dependentes cognitivos, foram representados pelos cuidadores responsáveis.

A identificação de necessidade de CP foi realizada pela escala Supportive and Palliative Care Indicators Tool - Versão em Português-BR (SPICT-BR) ${ }^{11}$, com análise de indicadores gerais de piora da saúde e de indicadores clínicos das condições avançadas, visando a identificação e avaliação da necessidade de CP para indivíduos com doenças crônicas sem possibilidade de cura, associado a deterioração progressiva da condição de saúde e risco de morte. Foram incluídos no estudo os pacientes idosos indicados por ACS, sem distinção de sexo, com idade superior a 60 anos, que apresentassem ao menos um indicador geral de piora da saúde e um indicador clínico de uma ou mais das condições avançadas, ou dois indicadores gerais de piora da saúde na escala SPICT-BR ${ }^{12}$.

Os idosos incluídos na pesquisa foram avaliados por meio de: Índice de Vulnerabilidade Clínico-Funcional-20 (IVCF-20) ${ }^{13}$, Questionário Sociodemográfico ${ }^{10}$, Escala de Sintomas de Edmonton (ESAS) ${ }^{14}$, Palliative Performance Scale (PPS $)^{15} \mathrm{e}$ da Palliative care Outcome Scale (POS) $)^{16-17}$.

Para a coleta de dados demográficos (sexo, idade), dados clínicos (tipo de doença limitadora de vida, tempo de diagnóstico, limitações funcionais), dados sociais e familiares (condição econômica, número de familiares no domicílio, característica do cuidador principal, entre outras) e dados do atendimento recebido pela UBS (tipo de cuidado, medicações, número de visitas realizadas pela equipe de saúde no mês, e limitações identificadas no atendimento), foi utilizado um questionário sociodemográfico adaptado de um estudo prévio ${ }^{10}$.

Foram excluídos pacientes que não atenderam aos critérios de avaliação propostos pelo protocolo pré-estabelecido para esta pesquisa, que mudaram do território ou evoluíram a óbito antes de seu início. A coleta de dados ocorreu por meio de atendimento individual para avaliação dos pacientes com possibilidade de deslocamento até a UBS e de visitas domiciliares para pacientes acamados e/ou domiciliados, acompanhadas por ACS de referência. Cada entrevista teve um tempo médio de aproximadamente uma hora e contou com a participação de uma aluna de graduação do curso de fisioterapia. 
Utilizou-se de estatística descritiva para análise quantitativa, com obtenção de médias e medidas de dispersão (desvio-padrão) para os dados numéricos e das frequências de dados categóricos, realizados com programa Excel 2013 (Microsoft). Os resultados foram apresentados por gráficos e tabelas.

\section{RESULTADOS}

A população inicial de estudo foi de 565 idosos cadastrados pelas equipes da ESF. Após uma primeira triagem realizada pelos ACS, foram excluídos 137 idosos $(24,2 \%)$ por não residirem mais na área de abrangência da UBS ou por terem falecido antes do início da pesquisa.

Foram incluídos 428 idosos para triagem inicial com a escala SPICT. Destes, 15 idosos (3,5\%) apresentaram dois ou mais indicadores de necessidade de CP e foram incluídos no protocolo de avaliação. Houve três (3) perdas (20\%), duas (2) por óbito antes da avaliação e uma (1) por mudança do território, totalizando 12 indivíduos investigados no presente estudo.

A média de idade dos participantes do estudo foi de 75 anos ( $D P=7,7)$, sendo sete mulheres e cinco homens. Cinco idosos $(41,7 \%)$ eram casados/as e cinco $(41,7 \%)$ viúvos/as, e a maioria $(66,7 \%)$ residia com mais pessoas e em sua residência própria $(66,7 \%)$. Onze idosos $(91,7 \%)$ eram aposentados ou com algum benefício social e não tinham planos privados de saúde (Tabela 1).

No que se refere aos cuidadores dos idosos, observou-se que seis eram esposos/as e quatro eram filhos/as, e a média de idade foi de 56,5 anos. Outros dados dos cuidadores são apresentados na tabela 1.

Tabela 1. Dados sociodemográficos de idosos com indicação de cuidados paliativos e seus cuidadores (Londrina, 2020).

\begin{tabular}{lcr}
\hline CARACTERISTICA & n & $\%$ \\
\hline Faixa etária & & \\
$>60$ anos & 4 & 33,3 \\
$>70$ anos & 5 & 41,7 \\
$>80$ anos & 3 & 25 \\
Sexo & & \\
Masculino & 5 & 41,7 \\
Feminino & 7 & 58,3
\end{tabular}




\section{Estado civil}

Casado/a

Solteiro/a

Divorciado/a

Viúvo/a

Quantidade de pessoas que convivem na residência

Nenhuma

$>1$

$>3$

Moradia

Própria

Moradia de familiar/cuidador

\section{Fonte de renda}

Seguridade Social

Suporte familiar

Plano de saúde privado

Sim

Não

Parentesco dos cuidadores

Esposo/a

Filho/a

Outros

Faixa etária dos cuidadores

$<59$ anos

$>60$ anos

Sexo dos cuidadores

Masculino

Feminino

Moradia dos cuidadores

Mesma do paciente

Outro domicílio

Fonte: Elaborado pelos autores (2020).

Com relação aos aspectos clínicos, verificou-se que entre as doenças em fase avançada, com características crônicas (conhecimento do diagnóstico maior que 2 anos), as mais frequentes eram as síndromes demenciais $(91,7 \%)$ seguido pelas doenças neurológicas (41,7\%). Nove idosos (75\%) apresentavam capacidade funcional reduzida e oito $(66,7 \%)$ dependiam de cuidadores para atender suas necessidades diárias (Tabela 2). 
Tabela 2. Aspectos clínicos de idosos com indicação de cuidados paliativos (Londrina, 2020).

\begin{tabular}{|c|c|c|}
\hline Condições clínicas & $\mathrm{n}$ & $\%$ \\
\hline \multicolumn{3}{|l|}{ Doença em fases avançada* } \\
\hline Síndrome demencial/ Fragilidade & 11 & 91,7 \\
\hline Doença neurológica & 5 & 41,7 \\
\hline Doença cardiovascular & 1 & 8,3 \\
\hline Condições clínicas & $\mathrm{n}$ & $\%$ \\
\hline \multicolumn{3}{|l|}{ Tempo de conhecimento da doença } \\
\hline$<6 \mathrm{~m}$ & 1 & 8,3 \\
\hline$>6 \mathrm{~m}$ & 2 & 16,7 \\
\hline$>1$ ano & - & - \\
\hline$>2$ anos & 9 & 75 \\
\hline \multicolumn{3}{|l|}{ Indicadores gerais de saúde } \\
\hline Internações recentes não programadas & 4 & 33,3 \\
\hline \multicolumn{3}{|l|}{ Tempo da última internação } \\
\hline$<1$ mês & 3 & \\
\hline$>1$ mês & - & \\
\hline$>3$ meses & 1 & \\
\hline$>6$ meses ou nenhuma & 8 & 66,7 \\
\hline Capacidade funcional ruim ou em declínio & 9 & 75 \\
\hline Dependente para cuidados pessoais & 8 & 66,7 \\
\hline $\begin{array}{l}\text { Perda de peso significativa nos últimos 3-6 meses ou } \\
\text { um baixo índice de massa corporal }\end{array}$ & 3 & 25 \\
\hline Sintomas persistentes apesar do tratamento otimizado & 1 & 8,3 \\
\hline $\begin{array}{l}\text { A pessoa ou família solicita cuidados paliativos, } \\
\text { limitação de tratamentos desconfortáveis ou foco na } \\
\text { qualidade de vida }\end{array}$ & 1 & 8.3 \\
\hline
\end{tabular}

${ }^{*}$ Admite mais de uma resposta

Fonte: Elaborado pelos autores (2020)

Ao avaliar a vulnerabilidade clínico-funcional, os 12 idosos foram considerados como frágeis pelo IVCF-20. Contudo, a avaliação clínico funcional realizada com a escala PPS, apontou pontuação média de 39 pontos, apenas um paciente obteve uma pontuação de $80 \%$, sendo que os demais pontuaram entre 30 a 50\% (Tabela 3 ).

Tabela 3. Avaliação funcional dos pacientes com indicação de cuidados paliativos (Londrina, 2020).

\begin{tabular}{cccc} 
PACIENTE & ESCORE IVCF-20 & CLASSIFICAÇÃO IVCF-20 & ESCORE PPS \\
\hline Paciente 1 & 33 & Frágil & 50 \\
Paciente 2 & 15 & Frágil & 40 \\
Paciente 3 & 30 & Frágil & 80 \\
Paciente 4 & 29 & Frágil & 30
\end{tabular}




\begin{tabular}{llll} 
Paciente 5 & 27 & Frágil & 30 \\
Paciente 6 & 20 & Frágil & 40 \\
Paciente 7 & 32 & Frágil & 50 \\
Paciente 8 & 19 & Frágil & 40 \\
Paciente 9 & 25 & Frágil & 30 \\
Paciente 10 & 17 & Frágil & 30 \\
Paciente 11 & 33 & Frágil & 40 \\
Paciente 12 & 33 & Frágil & 10 \\
\hline Média (DP) & $26,1(\mathrm{DP}=6,7)$ & & $39,2(\mathrm{DP}=16,8)$ \\
\hline
\end{tabular}

Fonte: Elaborado pelos autores (2020)

Com relação à intensidade dos sintomas, quando avaliados pela Escala de Avaliação de Sintomas de Edmonton, com variação de 0 a 10 em escala visual análoga, observou-se que todos os 12 idosos apresentavam o sintoma de dor, oito idosos referiram grau moderado ou grave (intensidade média 6,8). A falta de bem estar foi citado por 11 idosos (média de 6,0), cinco relataram o sintoma de cansaço (média 2,7), nove idosos também relataram alteração no sono com intensidade média de 5,0 e perda de apetite com intensidade média de 3,4. Além destes, outros sintomas citados foram depressão/tristeza (citado por sete idosos, média de 4,1), ansiedade (citado por seis idosos, média de 3,3), falta de ar (citado por quatro, média de 1,9) e náusea citado por um idoso.

$\mathrm{Na}$ figura 1, pode ser observada a distribuição dos sintomas nos idosos com indicação de CP, segundo grau de intensidade em leve, moderada ou intensa.

Figura 1. Distribuição dos sintomas entre idosos com necessidade de cuidados paliativos conforme o grau de intensidade.

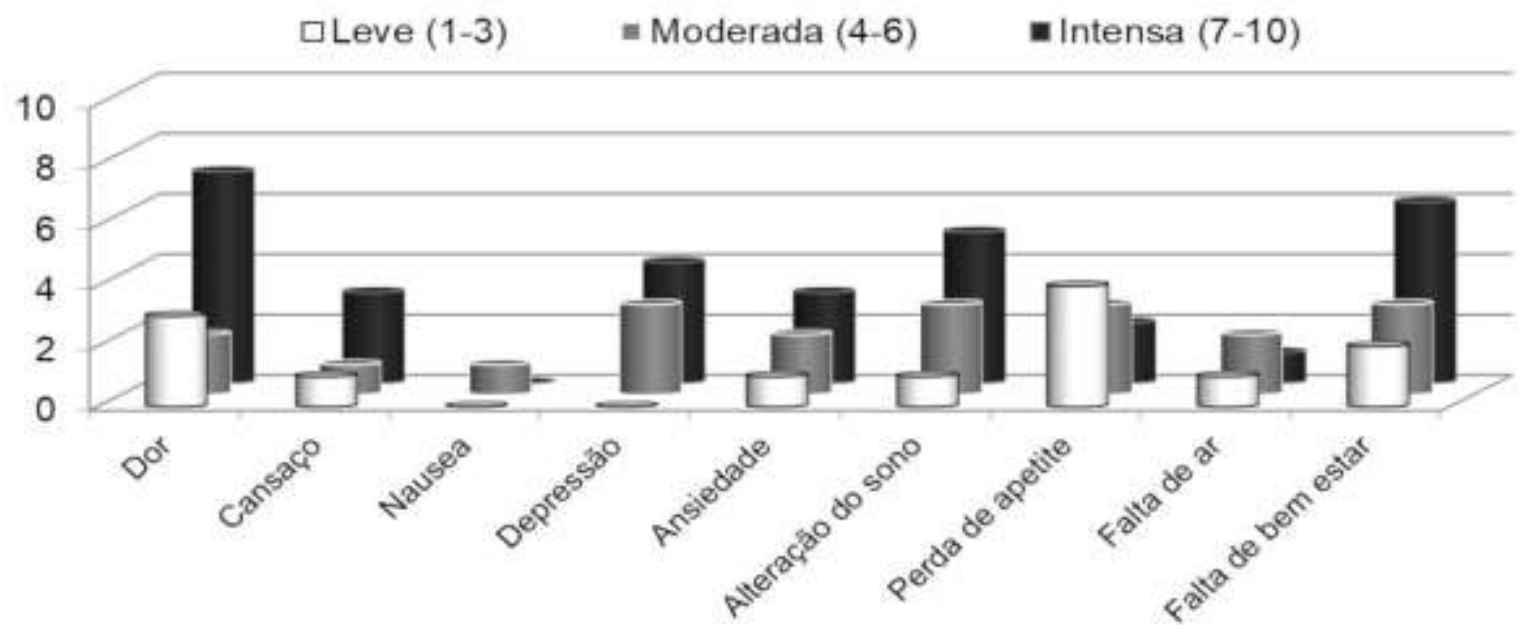

Fonte: Elaborado pelos autores (2020) 
No que se refere às principais demandas no contexto dos cuidados paliativos avaliadas pela ferramenta Palliative Outcome Scale (POS), no qual quanto maior a pontuação, maior o comprometimento do paciente, verificou-se uma média de 15,5 pontos $(\mathrm{DP}=6,3)$, com quatro respostas na versão do instrumento destinada a pacientes e oito na versão do cuidador.

Verificando a média de cada item da escala, nota-se que o item mais afetado foi a preocupação familiar, seguido da dor e do relato de sentir-se bem, tanto pelo paciente quanto pela percepção do cuidador. Apenas sete pacientes relataram que conseguiram compartilhar com a família e amigos como se sentiam, um paciente relatou que conseguiu compartilhar na maioria das vezes e quatro pacientes relataram não compartilhar nada ou compartilhar pouquíssimas vezes. Quando questionados quanto tempo gasto com compromissos relacionados com a saúde, a maioria referiu nenhum tempo gasto, dois referiram quase meio dia e dois referiram mais de meio dia gasto (Figura 2).

Figura 2. Distribuição da média por domínio da escala POS de idosos com necessidade de cuidados paliativos.

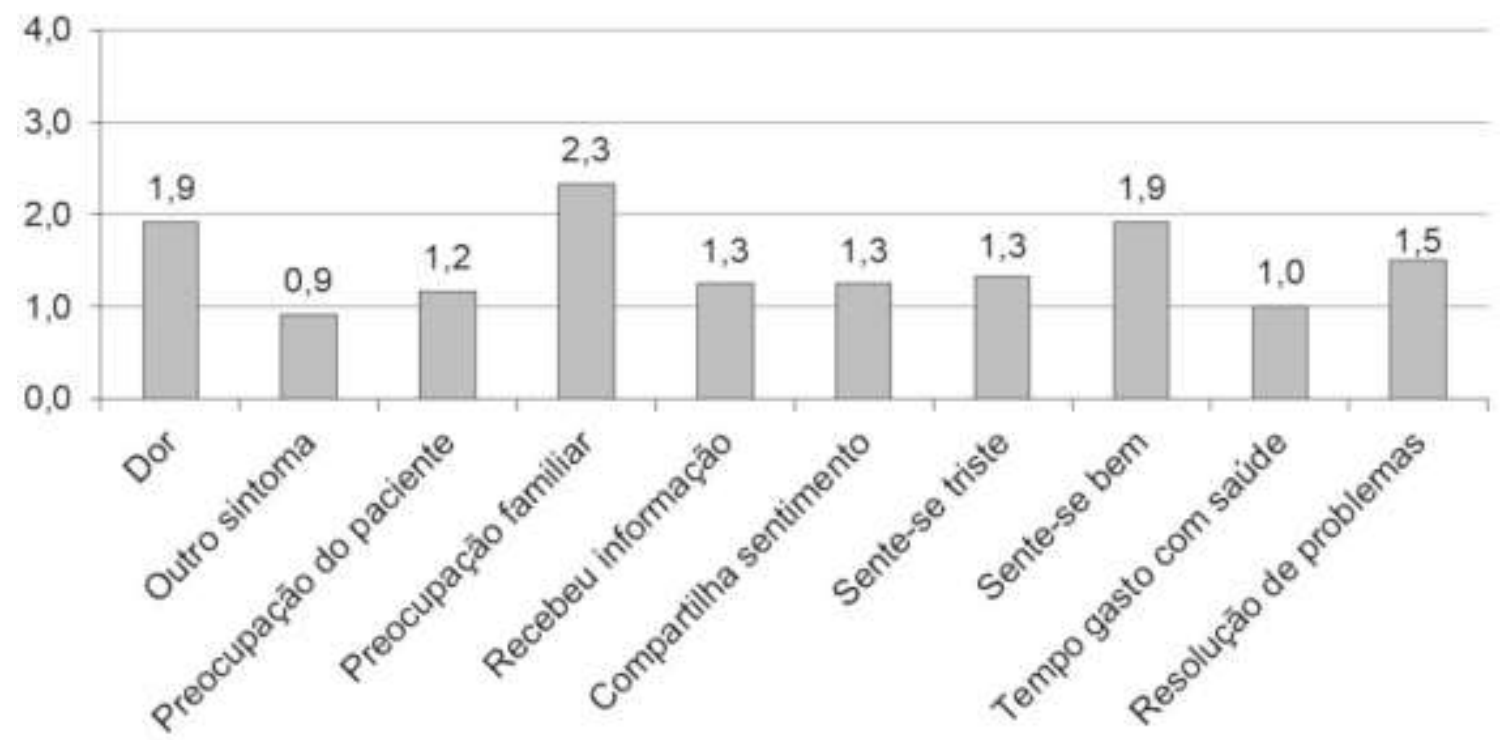

Fonte: Elaborado pelos autores (2020)

Sobre a assistência de saúde, verificou-se que nenhum idoso estava sob cuidados do serviço de atenção domiciliar (SAD). A maior parte dos idosos $(58,3 \%)$ utilizava a UBS para a obtenção de medicamentos e de atendimento de saúde. Sete $(58,3 \%)$ idosos relataram ter recebido visita de ACS no último mês, três $(25 \%)$ citaram a visita de fisioterapeuta neste período, dois $(16,7 \%)$ citaram visita da enfermagem e 
somente um $(8,3 \%)$ citou visita de médico. A maioria dos idosos referiu não ter problemas quanto ao serviço ofertado da UBS.

\section{DISCUSSÃo}

O presente estudo identificou pacientes idosos com necessidade de CP no contexto da ESF, acometidos principalmente por DCNT que afetam sua capacidade funcional, sendo necessário o controle dos sintomas que impactam sua qualidade de vida, por meio do atendimento de saúde prestado pela UBS onde residem. A APS tem o papel desafiador e imprescindível de conhecer o perfil do paciente que necessita de $\mathrm{CP}$, especialmente no que se refere aos aspectos inter-relacionais entre equipe de saúde, família e comunidade ${ }^{8-9}$.

Os achados sociodemográficos relacionados à faixa etária e sexo prevalente neste estudo corroboram com os achados em estudos semelhantes, no qual a faixa etária com maior incidência foi de 70 anos ou mais, sobressaindo-se o sexo feminino, com pequeno percentual $(3,6 \%)$ de pacientes na APS. Acredita-se que esse resultado possa estar relacionado à falta de avaliação e de acompanhamento na APS ${ }^{18}$.

Ainda sobre o perfil sociodemográfico da população de estudo selecionada, a maioria possuía casa própria, em geral eram aposentados ou com algum benefício social e usuários do sistema único de saúde (SUS) sem plano de saúde privado. A população da área de abrangência da UBS inserida neste estudo pode ser classificada como vulnerável, pois a área habitacional foi formada por beneficiários de programas de moradia popular e vinculados à assistência social. As moradias foram destinadas às famílias com baixa renda e, prioritariamente que moravam em áreas de fundo de vale, de proteção ambiental e/ou consideradas como irregulares no município. As famílias residiam no território à priori sem acesso a equipamentos sociais e públicos com 0 fator de vulnerabilidade e hábitos migratórios em comum entre os beneficiados $^{19}$.

Relativo aos aspectos clínicos encontrados, prevaleceram as síndromes demenciais e doenças neurológicas, com características crônicas e capacidade funcional em declínio. Estudos prévios apontam que no Brasil, em 2017, houve 30.662 óbitos por doenças cerebrovasculares e 2.173 óbitos por síndromes demenciais, entre 60 a 74 anos, com maior prevalência no sexo masculino ${ }^{20}$. As demências representam 
um dos principais distúrbios clínicos em idosos, gerando incapacidade funcional e sobrecarga de atenção e cuidados, tanto para as famílias quanto para os serviços de saúde $^{2}$. O European Sentinel Network Monitoring End-of-Life Care monitorou as causas de mortes em uma população acima de 65 anos na Bélgica, Itália e Espanha e verificaram como sendo a causa mais prevalente a demência ${ }^{21}$.

As síndromes demenciais e fragilidade foram os diagnósticos mais prevalentes entre os pacientes identificados para $\mathrm{CP}$ em uma pesquisa realizada com a população atendida pelo projeto "Estar ao Seu Lado - Cuidados Paliativos na Atenção Primária", no município de Rio Grande - Rio Grande do Sul. Os autores apontam ainda que no Brasil, não existe uma estratégia para a identificação de pacientes com necessidades de cuidados paliativos ${ }^{22}$.

Os CP dialogam com a abordagem multidisciplinar para produzir assistência de qualidade, voltada para o indivíduo sem possibilidade de cura, bem como para sua família, pois esta desenvolve um papel crucial como apoiador e facilitador da aceitação do diagnóstico e manejo do cuidado, e a responsabilidade e realização dos cuidados são frequentemente delegados às mulheres ${ }^{23-24}$. Neste estudo, observou-se a presença do cuidador para todos os indivíduos, que em sua maioria apresentaram dependência completa necessitando de maior suporte do cuidador. Ainda sobre o perfil dos cuidadores, houve predomínio do sexo feminino com grau de parentesco como esposas e filhas.

Os sintomas e sinais clínicos de maior prevalência nesta pesquisa foram dor, impactos na sensação de bem-estar, no sono e no apetite. Alguns idosos ainda apresentaram sintomas de depressão/tristeza e ansiedade, reafirmando que os aspectos psicossociais comprometem a qualidade da vida, tanto quanto os aspectos biofísicos, o que torna imprescindível a assistência multiprofissional ${ }^{10,25}$.

Algumas ferramentas podem ser utilizadas como tecnologias leve-duras para auxiliar a identificação dos pacientes com necessidade de CP e no julgamento clínico. Nesta pesquisa, foi utilizada para triagem inicial a SPICT-BR, validada em 2013 que possui tradução oficial para o português pelo site institucional dos criadores ${ }^{11,22,29}$. $O$ autor realizou um estudo para avaliar a aplicabilidade da escala SPICT-BR na prática clínica por equipes multiprofissionais em todos os níveis de atendimento com resultados positivos no Reino Unido e verificou que a ferramenta possibilita também a 
revisão e o planejamento antecipado do atendimento de pacientes com risco de deterioração e morte.

Com vistas a contemplar a multidimensionalidade da saúde do idoso e das síndromes geriátricas presentes no processo senil, em grande parte da população idosa, utilizou-se o Índice de Vulnerabilidade Clínico-Funcional-20 (IVCF-20), para avaliar os principais determinantes de saúde do idoso através de um questionário simples para profissionais não especialistas, para o próprio idoso ou sua família26,30.

Os idosos incluídos nesta pesquisa apresentaram indicadores de piora da saúde de uma ou mais das condições avançadas na triagem realizada com base na escala de SPICT-BR, e todos os participantes foram considerados como idosos frágeis, segundo avaliação realizada através da ferramenta IVCF-20.

Sobre a assistência recebida pela UBS e pelas equipes de saúde, verificou-se que nenhum paciente estava sob cuidado de serviço de atenção domiciliar (SAD) e que a maioria utilizava a UBS para a obtenção de medicamentos e de atendimento profissional. A ESF tem grande potencial para identificar e prestar cuidados paliativos básicos na APS, com cuidado centrado no usuário e sua família ${ }^{22,28}$. Tendo em vista os fortes vínculos da ESF com os pacientes, faz-se necessária a capacitação dos profissionais para oferecer alívio e conforto ao paciente com necessidade de cuidados paliativos, sendo imprescindível que os profissionais atuantes na UBS conheçam sua demanda e realizem as intervenções necessárias ${ }^{18,28}$.

Neste estudo os pacientes citaram poucas limitações sobre o suporte recebido da UBS referente aos cuidados com sua saúde. Entretanto, outros estudos identificaram a presença de "nós críticos" como a desarticulação entre os níveis assistenciais à saúde, falta de insumos e recursos humanos, ausência de capacitação dos profissionais e influências do modelo hospitalocêntrico e curativista na APS que dificultam o cuidado integral e longitudinal dos indivíduos e que se apresentam como um desafio que envolve a gestão para favorecimento de ações que visem o princípio da integralidade e ressignificação do cuidado no contexto domiciliar ${ }^{18,27}$.

É importante entender que os CP na APS não se aproximam da perspectiva de internação domiciliar, mas sim, visam garantir a especificidade de atendimento aos pacientes com menor complexidade, na perspectiva de controle de sintomas e 
planejamento do cuidado, e a referenciar aos demais níveis de atenção à saúde se necessário, buscando evitar a descontinuidade do cuidado ${ }^{24}$.

Este estudo revelou a vulnerabilidade do território e o hábito migratório dos usuários também como fator limitante, pois foram identificados pacientes que não permaneciam no domicílio semanalmente devido ao seu grau de dependência e indisponibilidade de cuidador e, muitos usuários $(24,2 \%)$ que não residiam no território, mas mantinham os domicílios cadastrados na área de abrangência da UBS.

Os resultados desta pesquisa demonstram um pequeno percentual de indivíduos idosos elegíveis para CP na população do território de abrangência da UBS. No entanto, ressalta-se que os óbitos registrados durante o período de realização desta pesquisa foram de três óbitos, ou seja, de 29,4\% dos pacientes selecionados na triagem inicial, que eram potenciais candidatos a inclusão no estudo, considerando as particularidades das condições de saúde dos pacientes elegíveis a $\mathrm{CP}$ e, que os indivíduos incluídos apresentavam tempo de conhecimento da doença maior que dois anos.

O envelhecimento populacional associado ao fenômeno de transição epidemiológica, com maior acometimento de DCNT aliado à falta de estratégias para a identificação de pacientes com necessidades de cuidados paliativos, desafiam a atenção básica e demandam maiores cuidados dos familiares e dos serviços de saúde, dificultando o acompanhamento e a intervenção precoce no que se refere ao enfrentamento desta condição no domicílio ${ }^{3,9}$. Os CP vão além da hegemonia hospitalar academicamente difundida, podendo também ser desenvolvido em ambiente ambulatorial e domiciliar. Contudo, é necessário maior número de pesquisas sobre CP no âmbito da APS ${ }^{9,22}$.

Sugere-se a realização de novos estudos para identificação e caracterização mais ampla de pacientes com indicação de cuidados paliativos nas APS, bem como a necessidade de investigação das políticas públicas vigentes e da relação e articulação entre as redes de cuidado no que se refere aos cuidados paliativos.

\section{CONCLUSÃo}

Observou-se no presente estudo que os idosos em situação de vulnerabilidade com necessidade de cuidados paliativos têm condições de fragilidade, baixa 
capacidade funcional e tem sua saúde e qualidade de vida impactadas pelas doenças crônicas. Os aspectos clínicos mais prevalentes foram as síndromes demenciais e doenças neurológicas, com características multifatoriais e capacidade funcional reduzida.

Todos os 12 idosos apresentavam, principalmente, os sintomas de dor, falta de bem estar, alteração no sono e perda de apetite, em alguns casos com intensidade moderada e alta. Além dos sintomas físicos, a preocupação familiar teve importante impacto na sensação de bem-estar dos pacientes. A maioria dos idosos utilizava a UBS para a obtenção de medicamentos e de atendimento de saúde e negou problemas quanto aos serviços ofertados.

A APS, como principal coordenadora do cuidado, tem alta potencialidade para promover integralidade e longitudinalidade para pacientes com necessidade de cuidados paliativos. Contudo, é necessário conhecer o perfil e as demandas destes pacientes, bem como, implementar políticas públicas de capacitação para as equipes de saúde, relacionadas aos CP no domicílio.

\section{REFERÊNCIAS}

1. Valcarenghi RV, Lourenço LFL, Siewert JS, Alvarez AM. Produção científica da Enfermagem sobre promoção de saúde, condição crônica e envelhecimento. Rev. Bras. Enferm [Internet]. 2015 [acesso em 14 out 2019]; 68(4): 705-12. doi: http://dx.doi.org/10.1590/0034-7167.2015680419i

2. Trelha CS, Dellaroza MSG. Cuidando do Idoso e sua família em situação de vulnerabilidade. Londrina: EDUEL, 2015.

3. World Health Organization. Saving lives, spending less: a strategic response to noncommunicable diseases. Geneva: WHO [Internet]. 2018 [Acesso em 09 abr 2020]. Disponível

em: https://apps.who.int/iris/bitstream/handle/10665/272534/WHO-NMH-NVI-18.8eng.pdf

4. Pessini L, Bertachini L. Novas perspectivas em cuidados paliativos: ética, geriatria, gerontologia, comunicação e espiritualidade. O mundo da saúde [Internet]. 2005 [Acesso em 09 abr 2020]; 29(4): 491-509. Disponível em: https://pdfs.semanticscholar.org/bb8b/ddc7effc8baf6af58d3190e86bbe872d3db1. pdf

5. Dalacorte RR, Rigo JC, Schneider RH, Schwanke CHA. Cuidados Paliativos em Geriatria e Gerontologia. São Paulo: Editora Atheneu, 2012. 384p. 
6. Malta DC, Bernal RTI, Lima MG, Araújo SSC de, Silva MMA da, Freitas MIF et al. Noncommunicable diseases and the use of health services: analysis of the National Health Survey in Brazil. Rev. Saúde Públ [Internet]. 2017 [Acesso em 09 abr 2020]; 51(1): 4s. doi: https://doi.org/10.1590/s1518-8787.2017051000090

7. Radbruch L, Lima L, Knau F, Wenk R, Ali Z, Bhatnaghar S, Blanchard C et. al. Redefining Palliative Cared: A New Consensus-Based Definition. J Pain Symptom Manage [Internet]. 2020 [acesso em 10 abr 2020]; 20(1). doi: https://doi.org/10.1016/i.jpainsymman.2020.04.027

8. Brasil. Ministério da Saúde. Portaria MS № 2.436, de 21 de setembro de 2017. Aprova a Política Nacional de Atenção Básica, estabelecendo a revisão de diretrizes para a organização da Atenção Básica, no âmbito do Sistema Único de Saúde (SUS). Brasília (DF): Ministério da Saúde [Internet]. 2017 [Acesso em 15 out 2019]. Disponível em: http://bvsms.saude.gov.br/bvs/saudelegis/gm/2017/prt2436 2209 2017.html

9. Sena GR. Cuidados Paliativos na atenção primária em saúde: estamos preparados para este desafio? In: Oliveira JT, Orange FA, Bezerra MR, Sena GR, Gusmão TL. Educação Permanente em Cuidados Paliativos: pauta para atenção primária. Recife: MIP, 2016. Cap. 1. p. 9-13.

10. Marcucci FCl. Identificação e caracterização dos indivíduos com indicação de cuidados paliativos cadastrados na estratégia saúde da família [Tese]. Londrina: Universidade Estadual de Londrina [internet]. 2016 [acesso em 13 out 2019]. 121 p.

em: http://www.bibliotecadigital.uel.br/document/?code=vtls000208832

11. Highet G, Crawford D, Murray SA, Boyd, K. Development and evaluation of the Supportive and Palliative Care Indicators Tool (SPICT): a mixed-methods study. BMJ Support Palliat. Care [Internet]. 2014 [acesso em 23 mar 2020];4(3):285-90. doi: http://dx.doi.org/10.1136/bmjspcare-2013-000488

12. Mitchell G, Senior H, Rhee J, Ware R, Young S, Teo P et al. Using intuition or a formal palliative care needs assessment screening process in general practice to predict death within 12 months: A randomised controlled trial. Pall Medicine [Internet]. 2017 [acesso em 14 abr 2020] ;32(2):384-94. doi: https://doi.org/10.1177/0269216317698621

13. Moraes E, Carmo J, Moraes F, Azevedo R, Machado C, Montilla D. ClinicalFunctional Vulnerability Index-20 (IVCF-20): rapid recognition of frail older adults. Rev. Mar Saúde Públ [Internet]. 2016 [acesso em 07 mar 2020]; 50(0). doi: https://doi.org/10.1590/S1518-8787.2016050006963

14. Monteiro DR, Almeida MA, Kruse MHL. Tradução e adaptação transcultural do instrumento Edmonton Symptom Assessment System para uso em cuidados paliativos. Rev Gaúcha Enferm [Internet]. 2013 [acesso em 12 abr 2020]; 34(2):163-171. Disponível em: http://www.scielo.br/pdf/rgenf/v34n2/v34n2a21.pdf

15. Victoria Hospice Society. Palliative Performance Scale (PPSv2). Victoria Hospice Society, Canadá [Internet]. 2009 [acesso em 16 mai 2020]. Disponível em: 
https://victoriahospice.org/wp-content/uploads/2020/08/PPSv2-QA-Instructionsand-Definitions-updated-July-2020.pdf

16. Correia FR. Tradução, adaptação cultural e validação inicial no Brasil da Palliative care Outcome Scale (POS). 2012. Dissertação (Mestrado em Enfermagem) Escola de Enfermagem de Ribeirão Preto, Universidade de São Paulo, Ribeirão Preto [Internet]. 2012 [acesso em 23 mar 2020]. doi: https://doi.org/10.11606/D.22.2012.tde-27032012-153946

17. Hearn J, Higginson IJ. Development and validation of a core outcome measure for palliative care: the palliative care outcome scale. Palliative Care Core Audit Project Advisory Group [Internet]. 1999 [acesso em 22 mar 2020]; v. 8, n. 4, 1999. Disponível em: https://www.ncbi.nlm.nih.gov/pmc/articles/PMC2483665/

18. Azevedo C, Rates C, Pessalacia J, Da Mata L. Perspectivas para os cuidados paliativos na atenção primária à saúde: estudo descritivo. Online Brazilian Journal of Nursing [Internet]. 2016 [acesso em 13 fev 2019]; 15(4):683. doi: https://doi.org/10.17665/1676-4285.20165370

19. Rizzotti MLA, Cordeiro SMA, Pastor M. Gestão de Políticas Sociais: território usado, intersetorialidade e participação. Londrina: Eduel, 2017.

20. Brasil. Ministério da Saúde. DATASUS. Estatísticas Vitais. Óbitos por causas evitáveis de 5 a 74 anos [Internet]. 2017 [acesso em 07 mar 2020]. Disponível em: http://tabnet.datasus.gov.br/cgi/deftohtm.exe?sim/cnv/evitb10uf.def

21. Miranda R, Penders Y, Smets T, Deliens L, Miccinesi G, Vega Alonso T et al. Quality of primary palliative care for older people with mild and severe dementia: an international mortality follow-back study using quality indicators. Age and Ageing [Internet]. 2018 [acesso em 20 ago 2019]; 47(6):824-33. doi: https://doi.org/10.1093/ageing/afy087

22. Corrêa SR, Mazuko C, Mitchell G, Pastrana T, De Lima L, Murray SA. Identifying patients for palliative care in primary care in Brazil: Project Estar ao Seu Lado's experience. Rev. Bras. Med. Fam. Comunidade [Internet]. 2017 [acesso em 13 fev 2020]; 12(39):1-8. doi: http://dx.doi.org/10.5712/rbmfc12(39)1507

23. Cardoso D, Muniz R, Schwartz E, Arrieira I. Cuidados paliativos na assistência hospitalar: a vivência de uma equipe multiprofissional. Texto \& Contexto - Enferm [Internet]. 2013 [acesso em 14 out 2019]; 22(4):1134-41. doi: https://doi.org/10.1590/S0104-07072013000400032

24. Sant' Ana L, D’Elboux M. Suporte social e expectativa de cuidado de idosos: associação com variáveis socioeconômicas, saúde e funcionalidade. Saúde em Debate [Internet]. 2019 [acesso em 25 mar 2020]; 43(121):503-519. doi: https://doi.org/10.1590/0103-1104201912117

25. Amaral JG. Medida de Independência Funcional de idosos portadores de doença crônica. 2010. 91 f. Dissertação (Mestrado em Enfermagem) - Universidade de Guarulhos, $\quad$ Guarulhos, 2010. Disponível em: http:/tede.ung.br/bitstream/123456789/229/1/Juliana+Gimenez+Amaral.pdf 
26. Carvalho GAFL, Menezes RMP de, Enders BC, Teixeira GA, Dantas DNA, Oliveira DRC de. Significados atribuídos por profissionais de saúde aos cuidados paliativos no contexto da atenção primária. Texto contexto - enferm [Internet]. 2018 [acesso em 04 mar 2020]; 27(2): e5740016. doi: https://doi.org/10.1590/0104-070720180005740016

27. Souza H, Zoboli E, Paz C, Schveitzer M, Hohl K, Pessalacia J. Cuidados paliativos na atenção primária à saúde: considerações éticas. Rev. Bioét [Internet]. 2015 [acesso em 20 mar 2020]; 23(2):349-59. doi: https://doi.org/10.1590/1983$\underline{80422015232074}$

28. Corrêa SR; Supportive and Palliative Care Indicators Tool (Brazilian version) [Internet]. Acesso em 15 jan 2019.Disponível em: https://www.spict.org.uk/thespict/spict-br/

29. Paraná. Secretaria de Estado da Saúde do Paraná. Avaliação multidimensional do idoso. Curitiba: SESA, 2018. 118 p. [acesso em 20 jan 2020]. Disponível em: https://www.saude.pr.gov.br/sites/default/arquivos restritos/files/documento/2020 -04/avaliacaomultiddoidoso 2018 atualiz.pdf

30. Marcucci F, Perilla A, Brun M, Cabrera M. Identificação de pacientes com indicação de Cuidados Paliativos na Estratégia Saúde da Família: estudo exploratório. Cad. Saúde Coletiva [Internet]. 2016 [acesso em 15 jan 2019]; 24(2):145-152. doi: https://doi.org/10.1590/1414-462X201600020012

RECEBIDO: 07/06/2021

ACEITO: 16/12/2021 